\title{
New Flavan and Benzil Isolated from Fissistigma latifolium
}

\author{
Yu-Hsuan Lan, ${ }^{* a}$ Yi-Ting Peng, ${ }^{a}$ Tran-Dinh Thang, ${ }^{b}$ Tsong-Long Hwang, ${ }^{c}$ Do-Ngoc Dai, ${ }^{d}$ \\ Yann-Lii Leu, ${ }^{c}$ Wan-Chun Lai, ${ }^{e}$ and Yang-Chang $\mathrm{Wu}^{*}, f$ \\ ${ }^{a}$ School of Pharmacy, China Medical University; ${ }^{f}$ Graduate Institute of Integrated Medicine, China Medical \\ University; Taichung 404, Taiwan: ${ }^{b}$ Department of Chemistry, Vinh University; Vinh City, Vietnam: ${ }^{c}$ Graduate \\ Institute of Natural Products, Chang Gung University; Tao-Yuan 333, Taiwan: 'Institute of Ecology and Biologycal \\ Resources, Vietnam Academy of Science and Technology; Vietnam: and ${ }^{e}$ Graduate Institute of Natural Products, \\ College of Pharmacy, Kaohsiung Medical University; Kaohsiung 807, Taiwan. \\ Received November 3, 2011; accepted December 1, 2011; published online December 6, 2011
}

Further investigation of the methanolic extract of Fissistigma latifolium resulted in two new compounds whose structures were assigned as 2,5,6,7-tetramethoxyflavan (1) and $2^{\prime}$-hydroxy-4', $5^{\prime}, 6^{\prime}$-trimethoxybenzil (2). These two compounds were determined on the basis of chemical and spectroscopic evidences. Compound 2 is the first report of benzil from Fissistigma species. 2,5,6,7-Tetramethoxyflavan (1) showed a potent inhibitory effect on superoxide anion production in formyl-L-methionyl-L-leucyl-L-phenylalanine (fMLP)/cytochalasin B (CB)-activated human neutrophils.

Key words Fissistigma latifolium; Annonaceae; flavan; benzil

Fissistigma genus (Annonaceae), consisting of about 90 species, has been shown to be a rich source of bioactive compounds. Until now, only about 16 species have been investigated for their constituents. ${ }^{1-12)}$ In literature, these species were reported to contain alkaloids, flavonoids, triterpenoids, benzenoids, cyclohexenones and cyclopentenones etc. ${ }^{1-12)}$ Bioactive components such as aristolactams from $F$. balance and $F$. oldhamii display antiplatelet aggregation activity; ${ }^{2)}$ kuafumine, fissistin and isofissistin from F. glaucescens and F. lanuginosum show potent cytotoxicity against oral epidermoid carcinoma cell in vitro. ${ }^{6,13)}$ Our previous investigation of Fissistigma species resulted in the isolation of alkaloids, furano-fissohamione, cyclopentenones and flavonoids., ${ }^{2,4,13-18)}$ Among these compounds, bracteolatum, piperoactam A, and fissislandione showed inhibitory effects on formyl-L-methionyl-L-leucyl-Lphenylalanine (fMLP)-induced superoxide anion $\left(\mathrm{O}_{2}{ }^{--}\right)$generation in human neutrophils and nitric oxide (NO) generation by RAW 264.7 macrophages in response to lipopolysaccharide (LPS). In the continuation of our search for constituents of $F$. latifolium, the isolation of two new compounds, 2,5,6,7-tetramethoxyflavan (1) and 2'-hydroxy-4',5',6'-trimethoxybenzil (2), is reported herein. The chemical structures of these two compounds were established by means of mass and related spectral experiments. These two compounds were also investigated for their effects on fMLP-induced superoxide anion $\left(\mathrm{O}_{2}^{--}\right)$generation in human neutrophils.

Compound 1 was obtained as a white amorphous solid. The molecular formula of compound $\mathbf{1}$ was determined to be $\mathrm{C}_{19} \mathrm{H}_{22} \mathrm{O}_{5}$ by high resolution-electron ionization-mass spectra (HR-EI-MS) ( $m / z$ 330.1479, Calcd 330.1467). IR spectrum indicated the presence of phenyl $\left(1612,1490 \mathrm{~cm}^{-1}\right)$ group. The UV spectrum exhibited maximal absorptions at 208 and $280 \mathrm{~nm}$. The ${ }^{1} \mathrm{H}-\mathrm{NMR}$ spectrum showed the presence of five aromatic protons at $\delta_{\mathrm{H}} 7.58(2 \mathrm{H}, \mathrm{d}, J=7.2 \mathrm{~Hz}), 7.43(2 \mathrm{H}, \mathrm{t}, J=7.2 \mathrm{~Hz})$, and $7.37(1 \mathrm{H}, \mathrm{d}, J=7.2 \mathrm{~Hz})$ of a mono-substituted benzene ring. Four protons of two adjacent methylene groups at $\delta_{\mathrm{H}}$ $1.79,2,27,2,67$ and 2.82 were evidenced by the correlation spectroscopy (COSY) and heteronuclear multiple quantum coherence (HMQC) spectrum. One singlet peak at $\delta_{\mathrm{H}} 6.49$ and three singlet peaks at $\delta_{\mathrm{H}} 3.80,3.85$, and 3.88 indicated the presence of one aromatic proton and three methoxy groups, respectively. The ${ }^{13} \mathrm{C}-\mathrm{NMR}$ spectrum showed nineteen carbon signals, including four methoxy, five methine, two methylene and seven quaternary carbons (Table 1). By comparison with literature data, ${ }^{19,20)}$ the above-mentioned information suggested a flavan skeleton. Heteronuclear multiple bond connectivity $(\mathrm{HMBC})$ correlations between $5-\mathrm{OCH}_{3}\left(\delta_{\mathrm{H}} 3.88\right) / \mathrm{C}-5$ $\left(\delta_{\mathrm{C}} 151.3\right), 6-\mathrm{OCH}_{3}\left(\delta_{\mathrm{H}} 3.80\right) / \mathrm{C}-6\left(\delta_{\mathrm{C}} 136.7\right)$, and $7-\mathrm{OCH}_{3}\left(\delta_{\mathrm{H}}\right.$ $3.85) / \mathrm{C}-7\left(\delta_{\mathrm{C}} 152.8\right)$ permitted the location of three methoxy groups at C-5, C-6, and C-7. The proton signal at $\delta_{\mathrm{H}} 6.49$ assigned to C-8 was due to its HMBC correlations between $\delta_{\mathrm{H}}$ 6.49 and $\delta_{\mathrm{C}} 108.8 / 136.7 / 148.8 / 152.8$ and the nuclear Overhauser effect spectroscopy (NOESY) cross peak between $\delta_{\mathrm{H}} 6.49$ and $\delta_{\mathrm{H}} 3.85\left(7-\mathrm{OCH}_{3}\right)$. The upfield methoxy group $\left(\delta_{\mathrm{H}} 3.02\right)$ connected to $\mathrm{C}-2\left(\delta_{\mathrm{C}} 100.4\right)$ was assigned by observing the HMBC correlation at $\delta_{\mathrm{H}} 3.02\left(2-\mathrm{OCH}_{3}\right), \delta_{\mathrm{H}} 2.27$ and $1.79(\mathrm{H}-3)$, and $2.67(\mathrm{H}-4 \mathrm{~b})$ with $\delta_{\mathrm{C}} 100.4(\mathrm{C}-2)$. The NOESY cross peaks between $\delta_{\mathrm{H}} 3.02\left(2-\mathrm{OCH}_{3}\right)$ and $\delta_{\mathrm{H}} 7.58\left(\mathrm{H}-2^{\prime}, \mathrm{H}-6^{\prime}\right)$ further confirmed this assignment. The structure of $\mathbf{1}$ was further confirmed by the mass spectrum. A prominent ion at $\mathrm{m} / \mathrm{z} 196$, consistent with a retro-Diels-Alder fragmentation, indicated that three methoxyl units were on ring A. Consequently, compound 1 was deduced to be 2,5,6,7-tetramethoxyflavan and is a new compound, reported here for the first time. Compound $\mathbf{1}$ is a racemate which was confirmed by the lack of Cotton effects in the circular dichroism (CD) spectrum.

Compound 2 was isolated as a yellow amorphous solid. The molecular formula, $\mathrm{C}_{17} \mathrm{H}_{16} \mathrm{O}_{6}$, was confirmed by HR-EI-MS ( $\mathrm{m} / \mathrm{z}$ 316.0955, Calcd 316.0947). The IR spectrum showed absorptions at 3404,1712 , and $1681 \mathrm{~cm}^{-1}$. The ${ }^{1} \mathrm{H}-\mathrm{NMR}$ protons at $\delta_{\mathrm{H}} 7.91(2 \mathrm{H}, \mathrm{d}, J=7.2 \mathrm{~Hz}), 7.63(1 \mathrm{H}, \mathrm{t}, J=7.2 \mathrm{~Hz})$, and 7.52 $(2 \mathrm{H}, \mathrm{t}, J=7.2 \mathrm{~Hz})$ showed the presence of a mono-substituted benzene ring. Three singlets at $\delta_{\mathrm{H}} 3.94,3.71$, and 3.60 indicated the presence of three methoxy groups. One downfield singlet at $\delta_{\mathrm{H}} 12.14$ revealed the presence of one hydrogen-bonded hydroxyl group. A singlet at $\delta_{\mathrm{H}} 6.29$ was due to an aromatic proton. The ${ }^{13} \mathrm{C}-\mathrm{NMR}$ spectrum showed seventeen carbon signals, including three methoxy, six methane and eight quaternary carbons. Among the eight quaternary carbons, two signals at $\delta_{\mathrm{C}} 190.9$ and 198.2 were identified as carbonyl 
<smiles>COc1cc2c(c(OC)c1OC)CCC(OC)(c1ccccc1)O2</smiles>

1

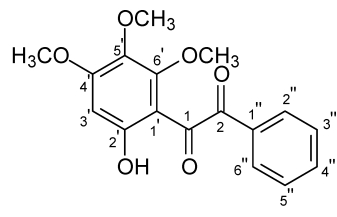

2
Fig. 1. Chemical Structures of Compounds $\mathbf{1}$ and $\mathbf{2}$

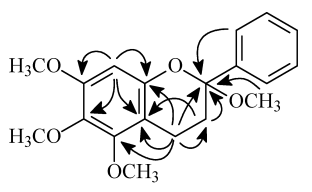

1

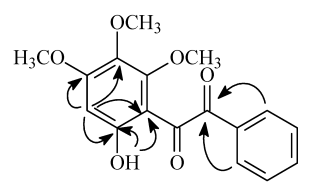

2
Fig. 2. Selected HMBC Correlations for $\mathbf{1}$ and $\mathbf{2}$

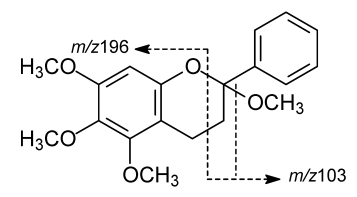

1

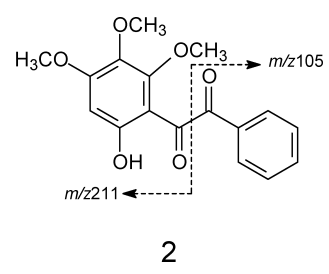

2
Fig. 3. Assignment of EI-MS Fragments for $\mathbf{1}$ and $\mathbf{2}$

groups (C-1, C-2). HMBC correlations at $\delta_{\mathrm{H}} 6.29$ with $\mathrm{C}-1^{\prime}$ $\left(\delta_{\mathrm{C}} 106.9\right), \mathrm{C}-2^{\prime}\left(\delta_{\mathrm{C}} 162.9\right), \mathrm{C}-4^{\prime}\left(\delta_{\mathrm{C}} 163.3\right)$ and $\mathrm{C}-5^{\prime}\left(\delta_{\mathrm{C}} 134.3\right)$ revealed that the proton was located at position $3^{\prime}$ and the NOESY cross peak between $\delta_{\mathrm{H}} 6.29$ and $\delta_{\mathrm{H}} 3.94\left(4^{\prime}-\mathrm{OCH}_{3}\right)$ further confirmed this assignment. Furthermore, the structure of compound 2 was deduced on the basis of the MS spectrum. Prominent fragment ions in the EI-MS of $\mathbf{2}$ were observed at $\mathrm{m} / \mathrm{z} 105$ and 211 which arose from rings $\mathrm{A}\left(\mathrm{C}_{10} \mathrm{H}_{11} \mathrm{O}_{5}\right)$ and $\mathrm{B}$ $\left(\mathrm{C}_{7} \mathrm{H}_{5} \mathrm{O}\right)$, respectively. Based on the NMR and MS spectra, the structure of $\mathbf{2}$ was established as $2^{\prime}$-hydroxy-4', $5^{\prime}, 6^{\prime}$ trimethoxybenzil. The natural occurrence of benzils is rare. For instance, 2,4,2-trihydroxy-4'-methoxybenzil was isolated from Zollernia paraensis and vijayosin from Pterocarpus marsupium. ${ }^{21,22)}$ Until now, no benzil has ever been reported from Fissistigma species. Compound $\mathbf{2}$ was the first benzil isolated from this species.

Two compounds were evaluated for their anti-inflammatory activities. The inhibitory activity of 2,5,6,7-tetramethoxyflavan (1) and 2'-hydroxy-4',5',6'-trimethoxybenzil (2) against the inflammatory response in human neutrophils was investigated. Compound 1 showed significant anti-inflammatory activity and inhibited the superoxide anion generation by $\mathrm{AMLP} / \mathrm{CB}$ induced human neutrophils, with $\mathrm{IC}_{50}$ values of $6.0 \pm 1.9 \mu \mathrm{M}$.

\section{Experimental}

General Experimental Procedures IR spectra were measured on a IRPrestige-21 spectrophotometer. ${ }^{1} \mathrm{H}-\mathrm{NMR}$ (400 MHz, using $\mathrm{CDCl}_{3}$ as solvent for measurement), ${ }^{13} \mathrm{C}-\mathrm{NMR}$ (100 MHz), HMQC, HMBC, ${ }^{1} \mathrm{H}-{ }^{1} \mathrm{H}$ COSY, distortionless enhancement by polarization transfer (DEPT), and NOESY spectra were recorded with Bruker Avance-400 NMR spectrometers. FAB-MS were collected on a JEOL JMS-SX/SX 102A mass spectrometer. EI-MS were collected on a MAT-95XL mass spectrometer. Silica gel 60 (Merck; 70-230, 230-400 mesh), sephadex LH-20 (GE healthcare) was used for column chromatography. TLC analysis was car-

Table 1. ${ }^{1} \mathrm{H}$ - and ${ }^{13} \mathrm{C}-\mathrm{NMR}$ Spectroscopic Data of Compounds $\mathbf{1}$ and $\mathbf{2}$

\begin{tabular}{|c|c|c|c|c|c|}
\hline \multicolumn{3}{|c|}{1} & \multicolumn{3}{|c|}{2} \\
\hline Position & $\delta_{\mathrm{H}}$ & $\delta_{\mathrm{C}}$ & Position & $\delta_{\mathrm{H}}$ & $\delta_{\mathrm{C}}$ \\
\hline & & & $1^{\prime}$ & & 106.9 \\
\hline 2 & & 100.4 & $2^{\prime}$ & & 162.9 \\
\hline $3 a$ & $\begin{array}{l}2.27(1 \mathrm{H}, \mathrm{dd} \\
\quad J=13.6,6.4 \mathrm{~Hz})\end{array}$ & 34.0 & $3^{\prime}$ & 6.29 & 96.3 \\
\hline $3 b$ & $\begin{array}{l}1.79(1 \mathrm{H}, \mathrm{ddd} \\
\quad J=13.6,6.4,2.4 \mathrm{~Hz})\end{array}$ & & $4^{\prime}$ & & 163.3 \\
\hline $4 a$ & $\begin{array}{l}2.82(1 \mathrm{H}, \mathrm{ddd}, \\
\quad J=16.4,11.6,6.4 \mathrm{~Hz})\end{array}$ & 16.6 & $5^{\prime}$ & & 134.3 \\
\hline $4 \mathrm{~b}$ & $\begin{array}{l}2.67(1 \mathrm{H}, \mathrm{ddd} \\
\quad J=16.4,6.2,2.4 \mathrm{~Hz})\end{array}$ & & $6^{\prime}$ & & 154.7 \\
\hline 5 & & 151.3 & $1^{\prime \prime}$ & & 133.3 \\
\hline 6 & & 136.7 & $2^{\prime \prime}, 6^{\prime \prime}$ & $7.91, \mathrm{~d}, J=7.2 \mathrm{~Hz}$ & 129.7 \\
\hline 7 & & 152.8 & $3^{\prime \prime}, 5^{\prime \prime}$ & $7.52, \mathrm{t}, J=7.2 \mathrm{~Hz}$ & 129.3 \\
\hline 8 & 6.49 & 97.2 & $4^{\prime \prime}$ & $7.63, \mathrm{t}, J=7.2 \mathrm{~Hz}$ & 134.4 \\
\hline 9 & & 148.8 & 1 & & 198.3 \\
\hline 10 & & 108.8 & 2 & & 190.1 \\
\hline $1^{\prime}$ & & 137.0 & $4^{\prime}-\mathrm{OCH}_{3}$ & 3.94 & 56.8 \\
\hline $2^{\prime}, 6^{\prime}$ & $7.58, \mathrm{~d}, J=7.2 \mathrm{~Hz}$ & 126.3 & $5^{\prime}-\mathrm{OCH}_{3}$ & 3.71 & 61.4 \\
\hline $3^{\prime}, 5^{\prime}$ & $7.43, \mathrm{t}, J=7.2 \mathrm{~Hz}$ & 128.4 & $6^{\prime}-\mathrm{OCH}_{3}$ & 3.60 & 60.9 \\
\hline $4^{\prime}$ & $7.37, \mathrm{t}, J=7.2 \mathrm{~Hz}$ & 128.3 & & & \\
\hline $2-\mathrm{OCH}_{3}$ & 3.02 & 49.4 & & & \\
\hline $5-\mathrm{OCH}_{3}$ & 3.88 & 60.1 & & & \\
\hline $6-\mathrm{OCH}_{3}$ & 3.80 & 60.5 & & & \\
\hline $7-\mathrm{OCH}_{3}$ & 3.85 & 55.5 & & & \\
\hline
\end{tabular}


ried out on $\mathrm{Si}$ gel $\mathrm{GF}_{254}$ pre-coated plates with detection using $50 \% \mathrm{H}_{2} \mathrm{SO}_{4}$ followed by heating on a hot plate.

Plant Material The plants of $F$. latifolium (Annonaceae) were collected from Phong Nha-Ke Bang National Park, Vietnam, in May, 2008, and the origin identified and authenticated by Dr. Tran Huy Thai (Institute of Ecology and Biological Resources, Vietnamese Academy of Science and Technology). A voucher specimen (20080515VN-TW) was deposited in the Herbarium of Vinh University, Vietnam.

Extraction and Isolation $F$. latifolium $(8.5 \mathrm{~kg})$ was extracted repeatedly with $\mathrm{MeOH}(15 \mathrm{~L} \times 3)$ at room temperature. The combined $\mathrm{MeOH}$ extracts were evaporated under reduced pressure to afford a syrup. The $\mathrm{MeOH}$ extracts $(186.6 \mathrm{~g})$ of $F$. latifolium were partitioned between EtOAc and $\mathrm{H}_{2} \mathrm{O}(1: 1, \mathrm{v} / \mathrm{v})$ to give an EtOAc fraction $(121.9 \mathrm{~g})$, which was then purified by column chromatography $(10 \times 25 \mathrm{~cm}$, Celite 545$)$ using $n$ hexane $(5 \mathrm{~L})$, followed by $\mathrm{CHCl}_{3}(5 \mathrm{~L})$, EtOAc $(5 \mathrm{~L})$ and $\mathrm{MeOH}$ $(10 \mathrm{~L})$ as elution solvents. This yielded 4 fractions ( $n$-hexane fraction $(\mathrm{EH}, 70.8 \mathrm{~g}), \mathrm{CHCl}_{3}$ fraction $(\mathrm{EC}, 39.7 \mathrm{~g})$, EtOAc fraction (EE, 2.5g) and a $\mathrm{MeOH}$ fraction (EM, 8.9g)). The $\mathrm{CHCl}_{3}$ fraction (EC, 39.8 g) was separated on a silica gel column $\left(8 \times 16 \mathrm{~cm}, \mathrm{CHCl}_{3}-\mathrm{MeOH}\right.$, gradient) to give 12 fractions. Fraction $5(8.1 \mathrm{~g})$ was purified on a silica gel column to afford 10 fractions. Subfraction 5-4 (2.7 g) was separated by silica gel column chromatography to provide 6 fractions. Subfraction 5-4-2 (1.3 g) was purified by repeated silica chromatography and then further purified twice by preparative thin layer chromatography to yield 2,5,6,7-tetramethoxyflavan (1) (7.5 mg) and 2'-hydroxy-4', $5^{\prime}, 6^{\prime}$-trimethoxybenzil (2) (3.2 mg).

Measurement of Superoxide Anion $\left(\mathrm{O}_{2}^{-}\right)$Generation The measurement of the generation of $\mathrm{O}_{2}{ }^{--}$was based on the superoxide dismutase(SOD)-inhibitable reduction of ferricytochrome $c .^{23)}$ In brief, after supplementing with ferricytochrome $c(0.5 \mathrm{mg} / \mathrm{mL})$, neutrophils $(6 \times 105 / \mathrm{mL})$ were equilibrated at $37^{\circ} \mathrm{C}$ for $2 \mathrm{~min}$ andincubated with either control or different concentrations of tested compounds for $5 \mathrm{~min}$. Cells were activated by fMLP $(0.1 \mu \mathrm{M})$ or PMA $(5 \mathrm{nM})$ for $10 \mathrm{~min}$. When fMLP was used as stimulant, cytochalasin B (CB, $1 \mu \mathrm{g}$ / $\mathrm{mL}$ ) was incubated for $3 \mathrm{~min}$ before peptide activation. The changes in absorbance with the reduction of ferricytochrome $c$ at $550 \mathrm{~nm}$ were continuously monitored in a double-beam, six-cell positioner spectrophotometer with constant stirring. Calculation is based on the difference of the reactions with and without SOD $(100 \mathrm{U} / \mathrm{mL})$ divided by the extinction coefficient for the reduction of ferricytochrome $c \quad(\varepsilon=21.1 /$ $\mathrm{mm} / 10 \mathrm{~mm})$.

2,5,6,7-Tetramethoxyflavan (1): White amorphous solid. UV $\left(\mathrm{CH}_{3} \mathrm{OH}\right) \lambda_{\max } \mathrm{nm}(\log \varepsilon): 208$ (4.20), 226 (3.64), 280 (3.17); IR $(\mathrm{KBr}) v_{\max } 2937,2833,1612,1593,1490,1465,1450,1413$, 1261, 1192, 1130, $1101 \mathrm{~cm}^{-1}$; ${ }^{1} \mathrm{H}-\mathrm{NMR}\left(\mathrm{CH}_{3} \mathrm{OH}, 200 \mathrm{~Hz}\right)$ : see Table $1 ;{ }^{13} \mathrm{C}-\mathrm{NMR}\left(\mathrm{CH}_{3} \mathrm{OH}, 50 \mathrm{~Hz}\right)$ : see Table 1; FAB-MS $\mathrm{m} / \mathrm{z}$ (rel. int. \%): $330\left(\left[\mathrm{M}^{+}, 7\right), 196\right.$ (8), 154 (100), 136 (73), 77 (22); HR-FAB-MS: $m / z=330.1479[\mathrm{M}]^{+}$, Calcd for $\mathrm{C}_{19} \mathrm{H}_{22} \mathrm{O}_{5}$.

2'-Hydroxy-4,5',6-trimethoxybenzil (2): Yellow amorphous solid. UV ( $\left.\mathrm{CH}_{3} \mathrm{OH}\right) \lambda_{\max } \mathrm{nm}(\log \varepsilon): 285$ (4.80), 340 (4.30); IR
(KBr) $v_{\max } 3404,3016,2927,2852,1712,1681,1610,1487$, 1450, 1350, 1298, 1259, 1240, 1205, $1109 \mathrm{~cm}^{-1}$; ${ }^{1} \mathrm{H}-\mathrm{NMR}$ $\left(\mathrm{CH}_{3} \mathrm{OH}, 200 \mathrm{~Hz}\right)$ : see Table $1 ;{ }^{13} \mathrm{C}-\mathrm{NMR}\left(\mathrm{CH}_{3} \mathrm{OH}, 50 \mathrm{~Hz}\right)$ : see Table 1; EI-MS (70 eV) m/z 316 [M] ${ }^{+}$(4), 211 (100), 105 (8), 77 (7); HR-EI-MS: $m / z=316.0955$ [M] $^{+}$, Calcd for 316.0947 .

Acknowledgement The investigation was supported by a Research Grant from the National Science Council of the Republic of China (NSC 97-2320-B-039-020-MY3) and a grant from China Medical University, Taichung, Taiwan (CMU100-TS-15).

\section{References}

1) Tran V. S., Trinh P. L., Trinh T. T., Nguyen T. H. A., Nguyen H. V., Tran D. Q., Nguyen T. A., Adv. Nat. Sci., 9, 55-67 (2008).

2) Chia Y. C., Chang F. R., Teng C. M., Wu Y. C., J. Nat. Prod., 63, $1160-1163$ (2000).

3) Yang Z., Niu Y., Le Y., Ma X., Qiao C., Phytomedicine, 17, 139141 (2010).

4) Lo W. L., Chang F. R., Wu Y. C., J. Chin. Chem. Soc., 47, 12511256 (2000).

5) Hao X., Lu Y., Shang L., Zheng Q., Chinese J. Med. Chem., 5, $143-145$ (1995).

6) Alias Y., Awang K., Hadi A. H., Thoison O., Sévenet T., Païs M., J. Nat. Prod., 58, 1160-1166 (1995).

7) Alias A., Hazni H., Jaafar F. M., Awang K., Ismail N. H., Molecules, 15, 4583-4588 (2010).

8) Chia Y. C., Wu J. B., Wu Y. C., Tetrahedron Lett., 41, 2199-2201 (2000).

9) Trinh T. T., Tran V. S., Nguyen T. H., Tạp Chí Hóa Học, 44, 412417 (2006).

10) Vichien J., Rapepol B., Porntip T., Rungruedee R., Kittisak L., Sci. Asia, 25, 31-33 (1999).

11) Liao Y. H., Guo J., Xu L. Z., Yang S. L., Acta Pharmacol. Sin., 34, 207-209 (1999).

12) Uddin M. J., Khan S. H., Mamun M. I. R., Nahar N., Aziz M. A., Mosihuzzaman M., J. Bangladesh Chem. Soc., 13, 175-179 (2000).

13) Wu Y. C., Lu S. T., Wu T. S., Lee K. H., Heterocycles, 26, 9-12 (1987).

14) Wu Y. C., Kao S. C., Huang J. F., Duh C. Y., Lu S. T., Phytochemistry, 29, 2387-2388 (1990).

15) Chia Y. C., Chang F. R., Li C. M., Wu Y. C., Phytochemistry, 48, 367-369 (1998).

16) Chia Y. C., Chang F. R., Wu Y. C., J. Nat. Prod., 61, 1430-1432 (1998).

17) Chia Y. C., Chang F. R., Wu Y. C., Tetrahedron Lett., 40, 75137514 (1999)

18) Lan Y. H., Chia Y. C., Chang F. R., Hwang T. L., Liaw C. C., Wu Y. C., Helv. Chim. Acta, 88, 905-909 (2005).

19) Lee D. Y., Kim D. H., Lee H. J., Lee Y., Ryu K. H., Jung B. I., Song Y. S., Ryu J. H., Bioorg. Med. Chem. Lett., 20, 3764-3767 (2010).

20) Ho J. C., Chen C. M., Phytochemistry, 61, 405-408 (2002).

21) Maurya R., Singh R., Deepak M., Handa S. S., Yadav P. P., Mishra P. K., Phytochemistry, 65, 915-920 (2004).

22) Ferrari F., Lima R. A., Bettolo G. B. M., Phytochemistry, 23, 2691-2692 (1984).

23) Hwang T. L., Su Y. C., Chang H. L., Leu Y. L., Chung P. J., Kuo L. M., Chang Y. J., J. Lipid Res., 50, 1395-1408 (2009). 